\title{
Molecular Infection in Tumor Growth Through Hypoxic Disorder
}

\author{
Iman Mostafa \\ Islamic Shiraz University, School of Veterinary Medicine, Shiraz, Iran
}

Correspondence Author: 1Iman Mostafa. Islamic Shiraz University, School of Veterinary Medicine, Shiraz, Iran

Received date: 11 June 2019, Accepted date: 28 July 2019, Online date: 8 August 2019

Copyright: (c) 2019 Iman Mostafa. This is an open-access article distributed under the terms of the Creative Commons Attribution License, which permits unrestricted use, distribution, and reproduction in any medium, provided the original author and source are credited.

\begin{abstract}
Hypoxia is a situation in which the body or part of the body is excluded of sufficient oxygen at the tissue level. And the Double-Stranded RNA virus is a powerful oncolytic virus, which is especially replicated in tumor cells and kills their result, called mammalian virus (MRV). This article investigates the ability of MRV to infect hypoxic tumor cells. This article showed that not only do they successfully translate proteins and replicate in hypoxic tumor cells, they also induce apoptosis. The paper also simplifies the involvement of any MRV protein in inhibiting HIF- $1 \alpha$ and obtaining $\mu 1$ and $\mu 2$ proteins capable of freely resetting HIF-1 $\alpha$. Prostate cancer interdependence (PCA) on androgens for growth and survival is elicited by hormone therapy, in which androgen deprivation is used as an appropriate treatment. However, initially successful, this androgenrelated PCA progresses to androgen-independent PCA, where hypoxia has been shown to play a critical role. The present study also investigated the effect of MRV infection on androgen-dependent hypoxic LNCaP cells. The results prove that MRV promotes apoptosis in hypoxic and normoxic LNCaP cells as well as activates Akt reality and does not regulate prostate-specific antigen (PSA).
\end{abstract}

Keywords: Molecular Infection, Hypoxic, Oxygen Modelling, Tumor Growth

\section{INTRODUCTION}

In general, hypoxia might be identified as general, affecting the whole body, or a locality affecting an area of the body. Although hypoxia is often a pathological disease, changes in arterial oxygen concentration are part of normal physiology, for example, during hypovolt nation training [1] or vigorous physical exercise. Hypoxia differs from anti hypoxemia and anoxemia because hypoxia refers to a condition where oxygen is insufficient, whereas hypoxemia and anoxemia are specifically referred to as those with low or zero arterial oxygenation. Hypoxia, where there is complete deprivation of oxygen, is called anoxia. Gradually symptoms of hypoxia levels, fatigue, numbness / tingling, nausea and cerebral anoxia [2-3]. These symptoms are usually difficult to identify, but priority Symptoms can be very important, which is called altitude sickness. In other words, hypoxia occurs when there is a reduced amount of oxygen in the body. Hypoxemia refers to depletion in $\mathrm{PO} 2$ below the normal range, regardless of whether gas exchange in the lung is impaired, $\mathrm{CaO} 2$ is adequate, or there is hypoxic tissue. There are several potential physiological mechanisms for hypoxemia, but in COP patients one of the most frequent cases is V/ Q mismatch, with or without alveolar hypoant nentation, as demonstrated by $\mathrm{PaCO}$. Hypoxemia due to $\mathrm{V} / \mathrm{Q}$ mismatch as seen in COPD, modification is relatively easy, as only small amounts of additional oxygen (less than 3 liters per minute for most patients) are required for LTOT. Although hypoxemia normally stimulates ventilation and causes dyspnea, these phenomena and other signs and symptoms of hypoxia deficiency are variable enough in patients with COPD and have limitations in evaluating patients.

Chronic alveolar hypoxia is a major contributor to right ventricular improvement of right ventricular hypertrophy with or without right ventricular failure in COPD patients. Negative pulmonary hypertension negatively affects COPD survival, to the extent that it increases the mean pulmonary artery pressure at rest. Although intensity of airflow blockage as measured by FEV1 is best correlated throughout prognosis in patients with COPD, chronic hypoxemia may increase intercourse and adverse effects for each disease severity. Extensive studies of LTOT in COP patients have shown a dose-response relationship between daily use of clock oxygen and survival.

One reason is that continuous, 24-hour oxygen consumption per day in appropriately selected patients provides an even greater survival advantage than has been demonstrated in NOTT and MRC studies. Strong tumor growth provides the presence of a local vascular network that supplies oxygen and nutrients to the tumor cells. However, a highly proliferating mass of tumor cells approaches the vasculature, and the tumor cells rapidly encounter an avascular environment of hypoxic, hypoxic. This is a consequence of the limit of oxygen release in tissues, measuring about $150 \mu \mathrm{m}[4,5]$. On histological examination, tumors often represent the central nucleus of necrotic cells, which has been suggested to be due to decreased oxygen availability to severe hypoxic conditions and glucose deprivation caused by cell death. A number of methods have been developed to measure oxygen concentration in tissues, including chemical makers such as pimonidazole hydrochloride or EF5, oxygen micro-electrodes or optical pressure partitions of oxygen measuring devices. Such areas of hypoxia have been hypothesized to decrease the response to radiation due to the reduction of oxygen-free radicals that are required to cause DNA damage. In addition, cells in these areas are resistant to biochemical therapy due to limited circulation of drugs. Hypoxic tumors also show extracellular $\mathrm{pH}$ (pHe), which is lower than the corresponding normal tissue. The nature of acidity is the result of changes in the metabolism of tumor cells, in particular glucose [5]. A significant number of genes involved in cellular metabolism, especially those that are glucose, are HIF-mediated. The cancers divert pyruvate metabolism away from oxidative phosphorylation of mitochondria 
(OXPHOS) to the cytoplasmic conversion of pyruvate to lactic acid [6-9]. Although this recent simplified pathway per glucose molecule decreases lid adenosine triphosphate (ATP), cells decrease the ATP production function by increasing the increase in glucose uptake and the flux in the conversion of glucose to pyruvate, i.e. glycolysis. This is possible by enhancing HIF-mediated expression in both glucose transporters and glycolytic pathway enzymes and gives tumors a "glycolytic" phenotype. Deviation of pyruvate to the lactate pathway through OXPHOS is also promoted by increased HIF-mediated expression of two key enzymes. Lactate Dehydrogenase A (LDH-A) and Pyruvate Dehydrogenase Ikinase 1 (PDK1) [5, 7, 10].

LDH-A is an enzyme that can convert pyruvate to lactate, and PDK1 is a pyruvate dehydrogenase inhibitor that feeds pyruvate into the tricarboxylic acid cycle and thus to OXPHOS. Thus, HIF not only pressurizes glucose to glycolysis and reduces mitochondrial respiration, but also calculates low respiration levels to the ratio of cytochrome c oxidase isoforms, components of the electron transport chain. Optimally slow [8]. The current work not only enhances respiration but may protect cells against oxidative damage under hypoxic deficiency. Metabolic regulation via HIF also provides products for tumor suppressors and endonucrons such as p53, c-Myc, Ras, and Akt [5-8]. Another pathway related to nutrient availability, as modified by HIF, is the mammalian rapamycin target (mTOR). On the one hand, growth factors and nutrients inhibit the mTOR pathway in transducing growth and survival signals by enhancing protein synthesis, and the other side reducing and hypoxia energy inhibits mTOR, saving energy-consuming protein synthesis, allowing cellular adaptation. And survival follows. [8].

\section{Hypoxia Tumor}

Initial study [1-3] using $\mathrm{O} 2$ oxygen needles in the clinical setting showed hypoxia in head and neck tumors. These authors have convincingly demonstrated that hypoxia in metastatic lesions with poor prognosis in radiotherapy is dissociated. The first data suggest that hypoxia can be a prognostic factor for patient income, published in [1]. In the first analysis of 31 patients with cervical cancer, the authors could show patients with hypoxic tumors (median pO2<10 mmHg) a significantly lower overall and relapse-free survival. These observations were confirmed in a follow-up study of 103 patients [2]. Survival differences were independent of stage, stage and stage rituals. Differences in local control in multivariate analysis were not apparent. Differences in survival were also observed in 106 patients at a cutoff of $\mathrm{PO} 2=5 \mathrm{~mm} \mathrm{HgIby} \mathrm{[3].} \mathrm{The} \mathrm{effect} \mathrm{of} \mathrm{hypoxia} \mathrm{in} \mathrm{this} \mathrm{recent} \mathrm{study} \mathrm{was} \mathrm{observed} \mathrm{only} \mathrm{in} \mathrm{patients} \mathrm{with} \mathrm{negative} \mathrm{nodules.} \mathrm{Again,} \mathrm{hypoxia}$ does not appear to be assessed by predictive control. Two small studies by [4] confirmed the impact of prognosis of hypoxia on disease-free survival and overall cervical cancer. Fruehauf [5] showed that hypoxia is a prognostic factor for local control (see also [5-7]) in contrast, a prospective international multicenter study [6] involving 120 patients with cervical cancer, obtained conflicting data without the effect of hypoxia. The reason for these inconsistent results is unclear. It also shows that hypoxia is prognostic for outcome in head and neck cancers, with data showing that hypoxia is a predictor for survival and local control. A central international study [8] of 397 patients with head and neck tumors provided further evidence that hypoxia with poor prognosis in patients with advanced. Head and Neck Cancer after Primary Radiation Therapy. In head and neck cancers, hypoxia not only predicts disease-free survival and overall survival (as it does in cervical cancer), but also localized topical control that promotes hypoxia radiation resistance. Predicts a major factor for local failure. Only a small number of patients were studied in the study, and hypoxia did not appear to be a prognostic factor in disease-free survival and local control. Studies of soft tissue sarcomas have also shown greater release of disease in patients with hypoxic tumors. In these studies, however, few patients permit multivariate analysis. For this reason, the effect of hypoxia on local control and remote control is unclear.

Mammalian Orth reovirus (MRV)

Abstract Mammalian orthovirus virus (MRV) is a double stranded RNA virus. It is part of the IReoviridae family, as well as the Spinareo virinae family. As the name implies, Ortheoreovirus mammals infect numerous species of mammals and vertebrates that act as natural hosts. Some of the diseases that result from or are associated with the virus include mild upper respiratory disease and gastrointestinal disease. Examples of these include upper respiratory tract syndromes, gastroenteritis (gastric influenza), biliary atresia, hydrocephalus obstruction, jaundice, alopecia, conjunctiva, and oily hair related to osteatria.

The name reovirus stands for respiratory enteric orphan virus.

Mammalian orthovirus obviously infects all humans with mammals and is essentially non-pathogenic [10], although there are some cases of pulmonary encephalitis in children, but the syndrome is rare but extremely rare. They are usually separated from the mammalian faeces as well as from fresh and salt sources. In general, there are three MRV serotypes based on neutralization and hemagglut nation profiles, type 1 lam (T1L), type 2 Jones (T2J), and type 3 dear (T3D), which differ mainly in their $\alpha 1$ substitution. In addition, one strain of serotype 3, strain Abney (T3A), was isolated from a child's anal swab suffering from gastrointestinal disease. MRV is considered after oral in ululation in newborn mice in M cells, and early infection occurs in the lymphoid tissue of Peyer patches before traveling to other sites such as the heart, brain, and liver. Figure 1 shows the virus organization. The figure shows shaded views of the surface of the re-viral virus, the intermediate subunit particle (ISVP), and the nucleus along with a cross-sectional view of each particle. And Figure 2 illustrate the MRV life cycle that Cell attachment occurs through $\sigma 1$.
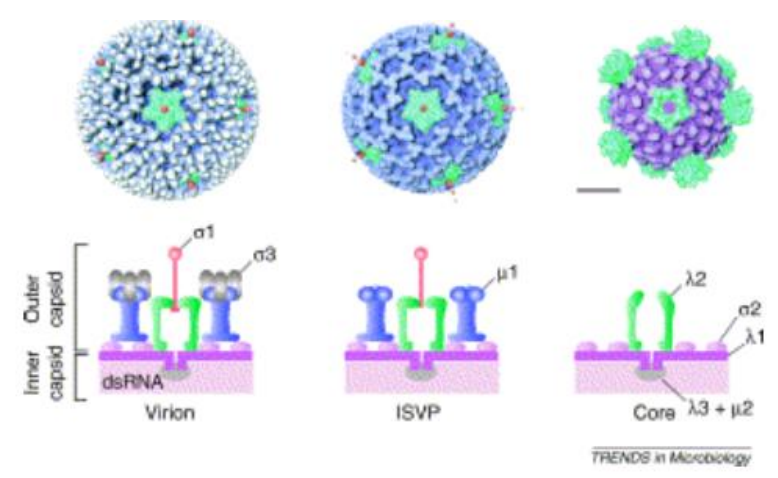

Fig. 1 virus particles Organization Image by Chandran et al [13].

\section{RESEARCH METHODOLOGY}

In the present paper, DU139 cells were observed in modified Pigeon extract (Invitrogen), PC3 cells were maintained in F-14K media, and LNCaP cells were maintained in RPMI medium (ATCC) containing $13.5 \%$ fetal serum and penicillin. Streptomycin (100 IU / ml, Mediatech). L839 cells maintained at minimum Joklik (Irvine Scientific) containing 1.6\% calf serum, 3.6\% fetal serum (Atlanta Biologicals), 3 mmol L-Glutamine (Mediatech) and penicillin-streptomycin (100 $\mathrm{IU} / \mathrm{ml}$, Mediatech). Were. All cell lines were obtained from the American Type Culture Collection.

The primary antibodies used were as follows: monolayer mouse anti-RACK2, rabbit monoclonal anti-HIF46 $1 \alpha$ rabbit anti- $\beta$ polyclonal antibody, rabbit antiPARP monoclonal antibody, rabbit polyclonal anti-micron rabbit [13], and mouse anti-HA. Secondary antibodies were used for immunodeficiency experiments: alkaline alkaline phosphatase (AP) goat anti-mouse or anti-rabbit IgG (Bio-Rad). Secondary antibodies were used in immunofluorescence experiments and date antibodies conjugated with Alexa 368 were used, and rabbit anti-rabbit and immunoglobulin G (IgG) antibodies nicknamed 985 (Invitrogen) were used. In case of infection, MRV Virions (strain T3D) is from the laboratory of Shiraz University. Pure virions as described were prepared using Freon's Vertrel reagent (DuPont) and placed in dialysis buffer $(130 \mathrm{mM} \mathrm{NaCl}, 16 \mathrm{mM}$ Tris pH $7.69 \div \mathrm{mm} \mathrm{MgCl})$ at $5^{\circ} \mathrm{C}$. Cells were seeded on $50 \mathrm{~mm}, 45 \mathrm{~mm}$, or $8.5 \mathrm{~cm} 2 \mathrm{cell} \mathrm{culture}$ dishes 2 $\mathrm{h}$ before $36 \mathrm{~h}$ injection.

In addition, a paper concludes with a numerical paper on the penetration of a spherical tumor by macrophages designed to secrete an oncolytic adenovirus under hypoxic environment. Numerical sales are made to predict the impact of therapeutic programs in which radiotherapy and engineered macrophages are separated. 


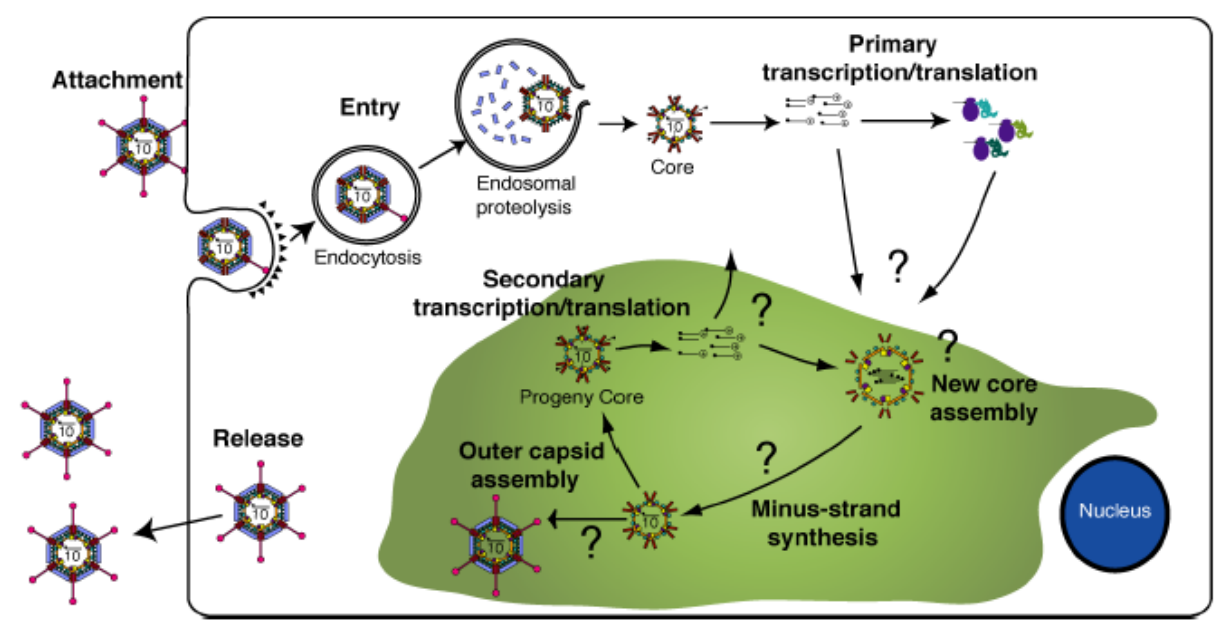

Fig. 2 the MRV life cycle

Hypoxic conditions were characterized by incubation at $0.9 \mathrm{O} \mathrm{O} 2$ and $7.5 \mathrm{CO} \mathrm{CO} 2$ at $36.8^{\circ} \mathrm{C}$ in a $23 \% \mathrm{CO} 2$ incubator equipped with a $23 \% \mathrm{CO} 2$ control. Before infection, all cells were incubated in hypoxia for $6 \mathrm{~h}$

Experiments were collected and subjected to 6 frost cycles. Ten-fold serial dilution was made in phosphate buffered saline (PBS) (146 mM calcium, $6 \mathrm{mM}$ calcium chloride, $9 \mathrm{mM} \mathrm{Na} 2 \mathrm{HPO} 4$ [pH 6.5]) containing $2 \mathrm{mM} \mathrm{MgCl} 2$ and plating units (PFU) were prepared using the method. The standard license plate was obtained on the L863. cells. The experiment was performed three times independently, but the mean of the four experiments plotted on a bar graph with error bars showed the standard error of the averages.

\section{RESULTS AND DISCUSSIONS}

\section{The MRV Infection}

Hypoxia-induced obstruction of negative protein synthesis negates the effect of some oncolytic viruses for reproduction in tumor cells growing in hypoxic environments. Since MRV elope clog body clog applied with disinfectant, [12, 14], this study predicts that MRV may reproduce in tumor cells growing in a hypoxic environment. To investigate the potential of the proposed hypothesis, this study identified three cell lines that represent androgen resistance, moderate I metastatic (DU139), androgen resistant, high metastatic (PC-3) and androgen-sensitive, Low metastatic (LNCaP) prostate. . In addition to growth in hypoxic environments, experiments were treated with cobalt chloride $(\mathrm{CoCl} 2)$, which mimics hypoxia in cells by inhibiting PHD2 hydroxylation of HIF-1 $\alpha$. This paper concludes that virus-induced protein translocation has not been altered by growth in hypoxic environments than in normoxic environments, suggesting that the virus is capable of inducing infection in tumor cells.

MRV infection leads to amplification of HIF-1 $\alpha$ proteasomes in colon cancer cells but increases HIF-1 $\alpha$ decay in brain tumor cells, suggesting that MRV infection differs in hypoxic cell response depending on the tumor type. Figure 3 Infection of MRV DU139, PC-2 and LNCaP infects or infects MRV T3D prostate tumor cells and is incubated in hypoxic environment $6 \mathrm{~h}$ before infection, $\mathrm{CoCl} 2$ also incubated with cells where they accumulate. Was. Figure 3-A indicates that the cells were harvested and proteins were separated on SDS-PAGE and transferred to nitrocellulose. Blots were performed with mouse polyclonal $\alpha-\mu \mathrm{NS}$ antiserum or mouse $\beta$-actin mouse polyconal antodiesodies followed by AP-conjugated sheep $\alpha$-mouse IgG antibody. Figure 1-B shows the cells at different times when the cells themselves harvested and were subjected to six freezing cycles. Standard virus plaque assay was performed on L653 cells on cell lysis to obtain virus titre (PFU). The mean PFU per milliliter per milliliter of submerged independent experiments, while error bars show a standard decrease.
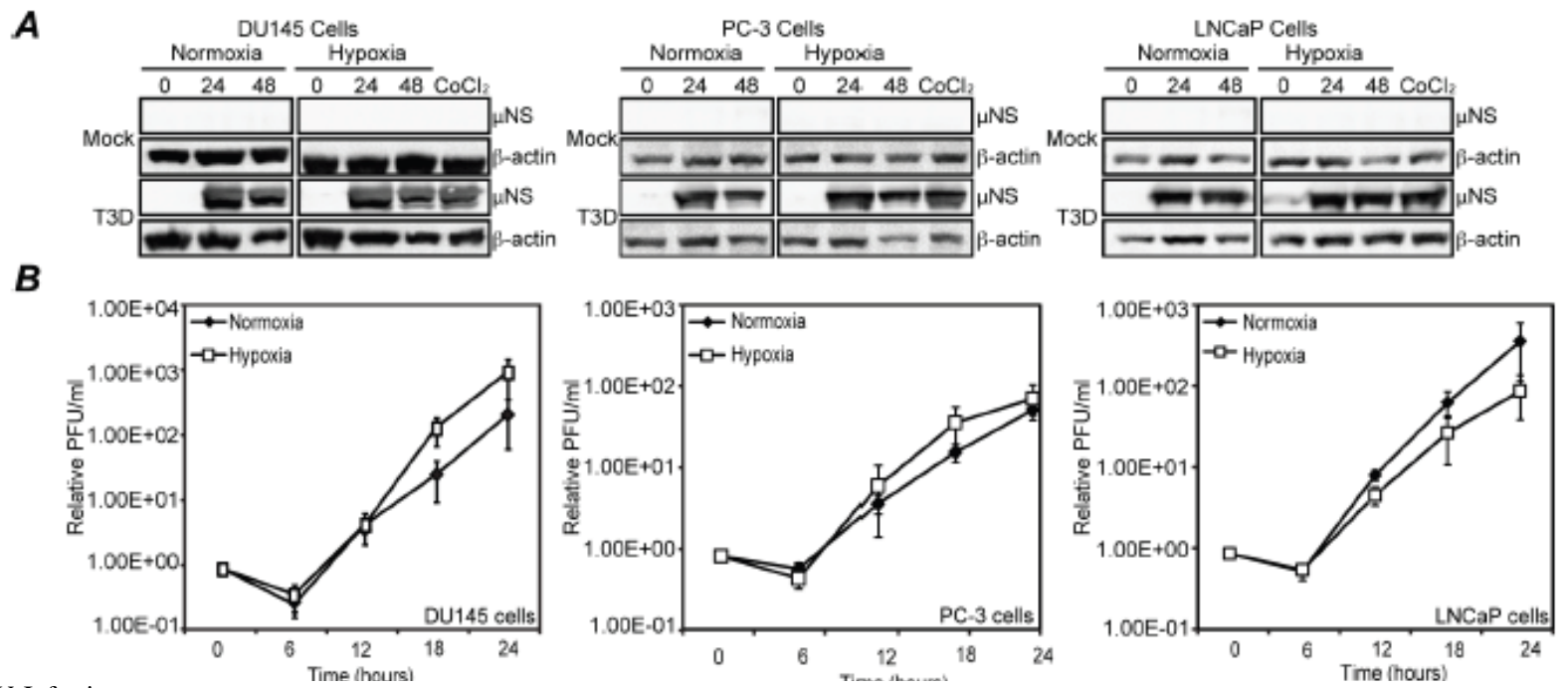

Fig. 3 MRV Infection.

To describe if the HIF-1 $\alpha$ statement was isolated by MRV infection in hypoxic tumor cells, the paper first performed an immunofluorescence test to test the HIF$1 \alpha$ statement at the individual cell surface. DU139, PC-3 and LNCaP cells were MRV infected or MRV infected and incubated in hypoxic medium. At $36 \mathrm{~h}$, cells were stained with anti-HIF-1 $\alpha$ antibodies. Figure 4 shows that DU139, PC3, and LNCaP cells were mock infected or MRV T3D infected. 48 hours before birth, the cells were fixed, with permeability and immunity to anti-NS antibody (red) and goat HIF-1 $\alpha$ antibody (green) followed by donkey Alexa Alexa 698- and mouse Alexa 398- Infrared corpses. An image merged with DAPI is shown. Strip, $10 \mathrm{~mm}$ 

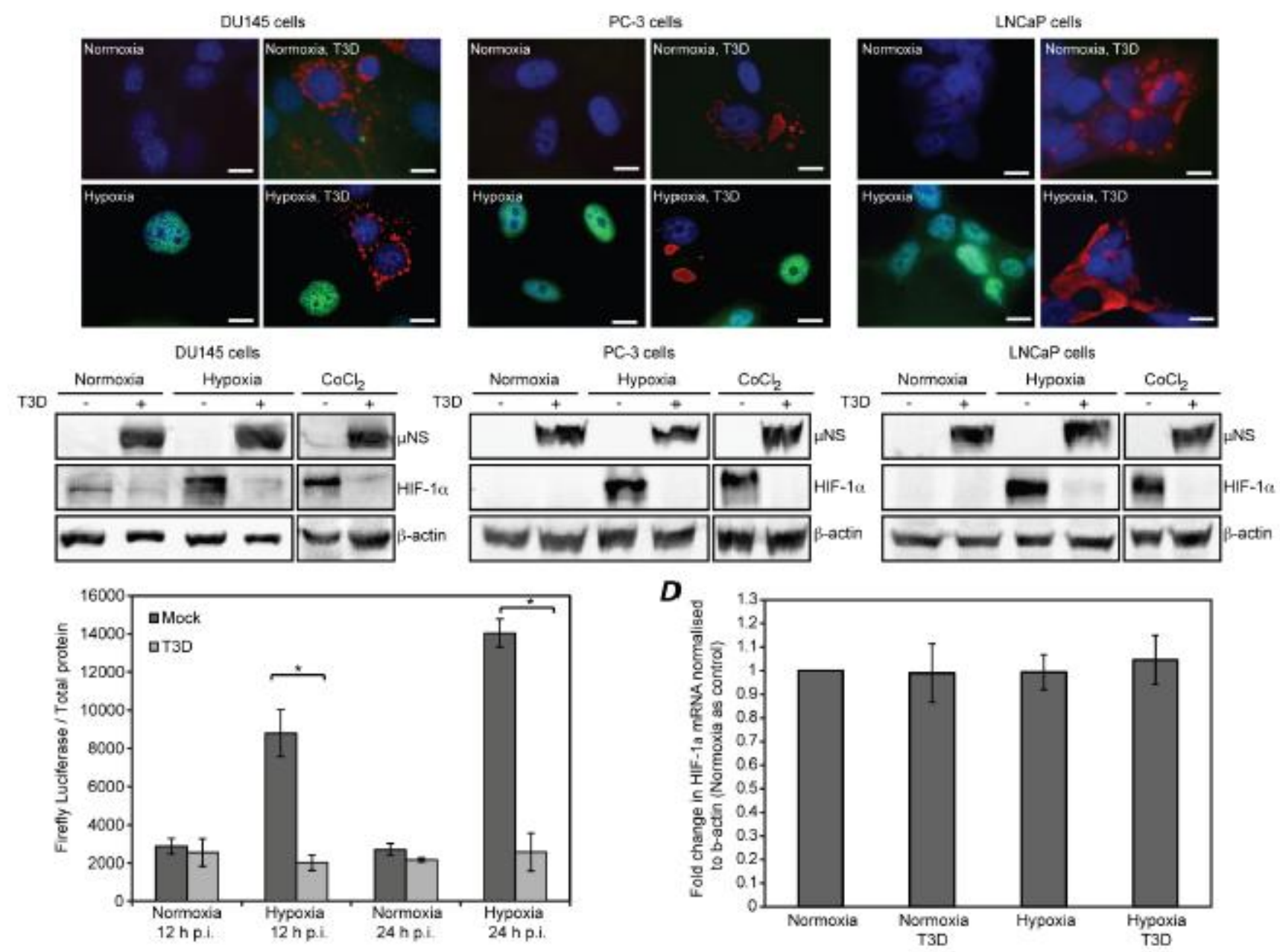

Fig. 4 Cells Contain Diminished HIF-1 $\alpha$

\section{THE NUMERICAL MODELLING}

In this paper, we have attempted to develop a numerical model for in vitro growth of aneurysm of aneurysmal vascular tumor that is infiltrated by macrophages. This article essentially follows the law [15-19] to obtain multi-stage tumor growth using mixed theory. The study also added another step for macrophages to follow the pathway [17-18].

The tumor is assumed to contain three stages of control: macrophages, tumor cells, and extracellular substances. While the model presented in [19] has assumed that all stages of a continuous continuous velocity step, the present paper introduces a specific velocity for each step. The governing equations are obtained by adding conservation of mass andimomentum to each step. The model closes by establishing constructive assumptions about the material properties of each step, the interaction between the steps and the factors that influence cell proliferation and death.

Applying the principle of mass equilibrium in the three element phases, $1, \mathrm{~m}$ and $\mathrm{n}$, provides the sources.

$\partial \mathrm{l} \partial \mathrm{t}=\mathrm{Sl}-\nabla \cdot(\mathrm{lvl})$

$\partial \mathrm{m} \partial \mathrm{t}=\mathrm{Sm}-\nabla \cdot(\mathrm{mvm})$

$\partial \mathrm{n} \partial \mathrm{t}=\mathrm{Sn}-\nabla \cdot(\mathrm{nvn})$

In Eq 1, 2 and 3 the functions $S_{l}, S_{m}$, and $S_{n}$ represent the net rates of production for each phase. Equations for $\mathbf{v}_{l}, \mathbf{v}_{m}$, and $\mathbf{v}_{n}$ are derived by applying conservation of momentum to each phase and neglecting inertial effects:

$0=\nabla \cdot(l \sigma l)+F l n+F l m+P \nabla l+F a$

$0=\nabla \cdot(\mathrm{mom})+\mathrm{Fmn}+\mathrm{Fml}+\mathrm{P} \nabla \mathrm{m}$

$0=\nabla \cdot(\mathrm{n} \sigma \mathrm{n})+\mathrm{Fnm}+\mathrm{Fnl}+\mathrm{P} \nabla \mathrm{n}$

In Eq. 4, 5 and 6 the first term represents the internal forces in each phase while $\mathbf{F}_{i j}(\mathrm{i}, \mathrm{j}=1, \mathrm{~m}, \mathrm{n} ; \mathrm{i} \neq \mathrm{j})$ denotes the inter-phase force exerted on phase $j$ by phase $i$, noting that $\mathrm{Fij}=-\mathrm{Fji}$.

Following the root of [20] the functions $d_{l}(c)$ and $d_{m}(c)$ model the rate at which macrophages and tumor cells die due to lack of oxygen.

$\mathrm{dl}(\mathrm{c})=\mathrm{d}^{\wedge} 1[1-\beta(\mathrm{c}, \mathrm{cc})]$

$\mathrm{dm}(\mathrm{c})=\mathrm{d}^{\wedge} \mathrm{m}[1-\beta(\mathrm{c}, \mathrm{cc})]+\mathrm{k}^{\wedge} \mathrm{l}[1-\beta(\mathrm{c}, \mathrm{cp})]$

Where both CC and CP are stable oxygen thresholds. The positive scales of d) $\mathrm{m}$ and $\mathrm{d}^{\wedge} \mathrm{m}$, respectively, are the death rates of macrophages and tumor cells. $\mathrm{k}=$ constant measures the rate of death in which macrophages lyse hypoxicomor cells. The following article presents new boundary environments for macrophages and chemotherapy on the other side of the $r=R(T)$ boundary. The paper also assumes that the flux of each step on the other side is the dispersal boundary, proportional to the various of field between the phase observed at the boundary and the phase concentration in the culture medium surrounding the tumor (assumed 
correction during analysis). In addition, the tumor boundary is permeable to oxygen and thus produces a Dirichlet boundary and stabilizes the oxygen concentration. By combining the above assumptions it gives us.

$-\mathrm{l}(\mathrm{vl}-\mathrm{vm})=\mathrm{hl}(\mathrm{l} \infty-1)-\mathrm{n}(\mathrm{vn}-\mathrm{vm})=\mathrm{hn}(\mathrm{n} \infty-\mathrm{n}) \mathrm{c}=\mathrm{c} \infty \partial \mathrm{a} \partial \mathrm{r}=\mathrm{ha}(\mathrm{a} \infty-\mathrm{a})\}$ onr $=\mathrm{R}(\mathrm{t})$

$\mathrm{m}(\mathrm{r}, 0)=\mathrm{m} 0,1(\mathrm{r}, 0)=0, \mathrm{c}(\mathrm{r}, 0)=\mathrm{c} \infty, \mathrm{a}(\mathrm{r}, 0)=0, \mathrm{R}(0)=\mathrm{R} 0$

$\mathrm{vm}=-1 \mathrm{k}(\partial \mathrm{P} \partial \mathrm{r}+\mathrm{Dmm} \partial \mathrm{m} \partial \mathrm{r})$

The hl, hn and ha constants indicate tumor permeability to macrophages, extracellular materials, and chemotherapy, respectively. It is also recommended that the tumor cell size decrease be spatially different at each point for 0 degrees and that the tumor is not initially isolated from macrophages and chemistries.

To construct a numerical modeling of the governing relationships, it is appropriate to first introduce a (r, t) $\rightarrow(r R(t), t)$ coordinate change that addresses the moving boundary problem on a fixed domain (Figure 7-9, 15). -17 for further reading). The obtained equations are then obtained using the lines method: approximation of finite element difference for the interpretation of spatial derivatives to 900 grid points and a regular time-dependent ordinary differential equation system using a stepwise, rank order variable. Has been achieved. Fig. 5 shows the radius measurement over a period of time of HIF-1 $\alpha$ tumor spheroids in which each measurement sink had an average radius of 30 spheroids grown in the absence of macrophages (black, top and right axes shown). And $\mathrm{R}$ is a dimensionless (t) curve when determined by Eqs. (1) - (3) and (8) - (9) are obtained using said parameters first. Figure 6 shows the spatial distribution of tumor cells and the oxygen concentration in the model solution in relation to the tumor radius map $\mathrm{R}(\mathrm{t})$ shown above.

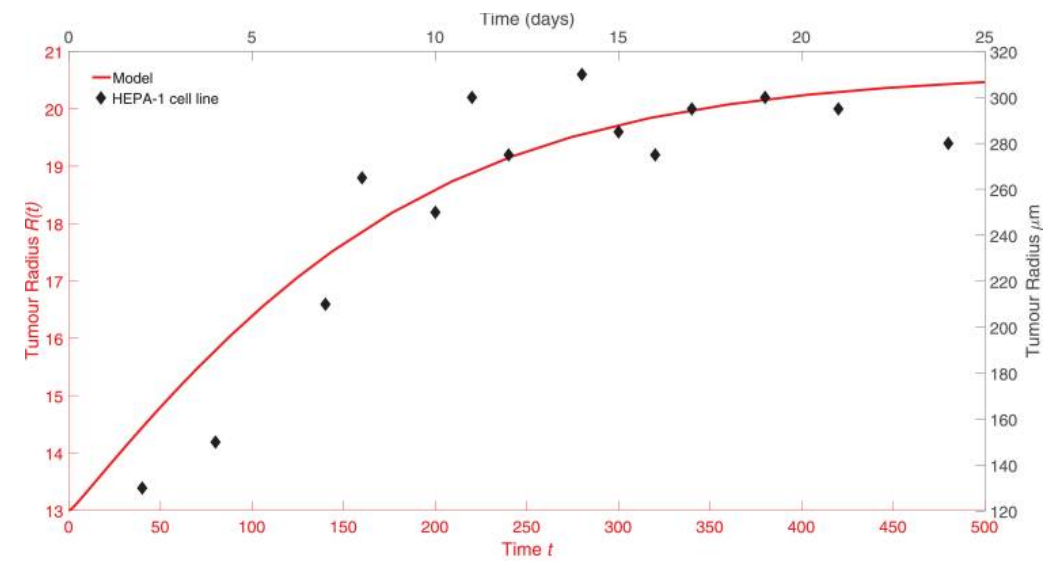

Fig. 5 Radius Measurements
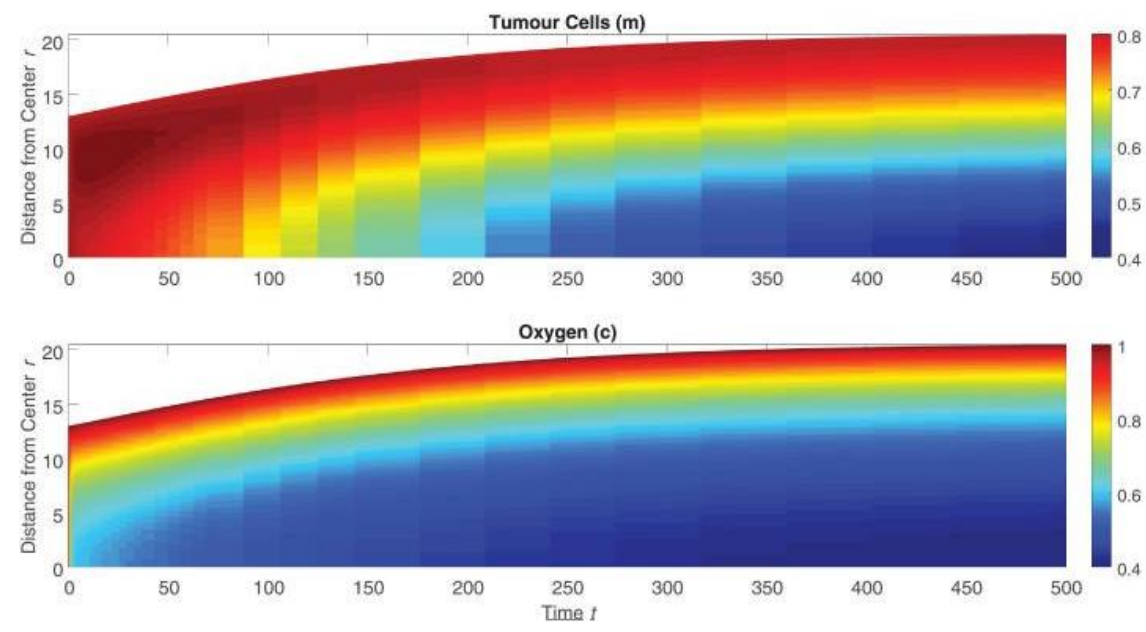

Fig. 6 The Spatial Distribution of the Tumor Cells and Oxygen

The first two terms of efficacy, first mentioned, are essential for the release of oncolytic virus while macrophages undergo hypoxia and release when cells die after being infected with the tumor. The positive P parameter contains the virus release rate from engineered macrophages in hypoxic environments, and measures the scale constant of the amount of virus released by the death of infected cells. Unexpected tumor cells can become infected by contact with the oncolytic virus. By comparison with the following formulas, the volume fraction of uninfected cells is dominant.

$\partial \mathrm{l} \partial \mathrm{t}=1 \mathrm{r} 2 \partial \partial \mathrm{r}[\mathrm{r} 2 \mathrm{k}(\mathrm{Dl}(1-1) \partial \mathrm{l} \partial \mathrm{r}-1 \mathrm{Dm}(\partial \mathrm{mU} \partial \mathrm{r}+\partial \mathrm{mI} \partial \mathrm{r})-\chi \mathrm{l}(1-1) \partial \mathrm{a} \partial \mathrm{r})]-1 \mathrm{dl}(\mathrm{c})$

$\partial \mathrm{mI} \partial \mathrm{t}=1 \mathrm{r} 2 \partial \partial \mathrm{r}[\mathrm{r} 2 \mathrm{k}(\mathrm{Dm}(\partial \mathrm{mI} \partial \mathrm{r}-\mathrm{mI}(\partial \mathrm{mU} \partial \mathrm{r}+\partial \mathrm{mI} \partial \mathrm{r}))-\mathrm{mIDl} \partial \mathrm{l} \partial \mathrm{r}+\chi 1 \mathrm{mI} \partial \mathrm{a} \partial \mathrm{r})]$

$\mathrm{r} \phi \phi \mathrm{mU}-\mathrm{mI}[\mathrm{dm}(\mathrm{c})+\mathrm{lk}(\mathrm{c})+\mathrm{k} \phi], \partial \mathrm{mU} \partial \mathrm{t}=1 \mathrm{r} 2 \partial \partial \mathrm{r}[\mathrm{r} 2 \mathrm{k}(\mathrm{Dm}(\partial \mathrm{mU} \partial \mathrm{r}-\mathrm{mU}(\partial \mathrm{mU} \partial \mathrm{r}+\partial \mathrm{mI} \partial \mathrm{r}))-\mathrm{mUDl} \partial \mathrm{l} \partial \mathrm{r}+\chi \mathrm{lmU} \partial \mathrm{a} \partial \mathrm{r})]$

$\mathrm{mU}[\mathrm{pm}(\mathrm{mU}+\mathrm{mI}, \mathrm{c})-\mathrm{dm}(\mathrm{c})-\mathrm{k}(\mathrm{c})-\mathrm{r} \phi \phi]-\mathrm{R}(\mathrm{mU}, \mathrm{ts}), \partial \mathrm{c} \partial \mathrm{t}=1 \mathrm{r} 2 \partial \partial \mathrm{r}(\mathrm{r} 2 \mathrm{Dc} \partial \mathrm{c} \partial \mathrm{r})-\mathrm{d}^{\wedge} \mathrm{clcl}-\mathrm{d}^{\wedge} \mathrm{cmc}(\mathrm{mU}+\mathrm{mI})$

$\mathrm{d}^{\wedge} \mathrm{cp}(\mathrm{mU}+\mathrm{mI}) \mathrm{pm}(\mathrm{mU}+\mathrm{mI}, \mathrm{c}), \partial \mathrm{a} \partial \mathrm{t}=1 \mathrm{r} 2 \partial \partial \mathrm{r}(\mathrm{r} 2 \mathrm{Da} \partial \mathrm{a} \partial \mathrm{r})+\gamma \mathrm{R}(\mathrm{mU})$

$[1-\beta(\mathrm{c}, \mathrm{cp})]\left[\mathrm{p}^{\wedge} \mathrm{all}+\mathrm{p}^{\wedge} \mathrm{am}(\mathrm{mU}+\mathrm{mI})\right]-\lambda \mathrm{aa}, \partial \phi \partial \mathrm{t}=1 \mathrm{r} 2 \partial \partial \mathrm{r}(\mathrm{r} 2 \mathrm{D} \phi \partial \phi \partial \mathrm{r})+\mathrm{lp} \mathrm{p}^{\wedge} \phi[1-\beta(\mathrm{c}, \mathrm{cc})]$

$\eta[\mathrm{dm}(\mathrm{c}) \mathrm{mI}+\operatorname{lmIk}(\mathrm{c})+\mathrm{k} \phi \mathrm{mI}]-\phi(1-\beta(\mathrm{mI}+\mathrm{mU}, \mathrm{c} \phi))$

In this paper, the above equations are simulated and the results show that both the treated and the macrophage viruses are treated simultaneously, thus attempting to measure the size of the infected cells using either radiotherapy or macrophage treatment alone. - Reduce viral therapy. 


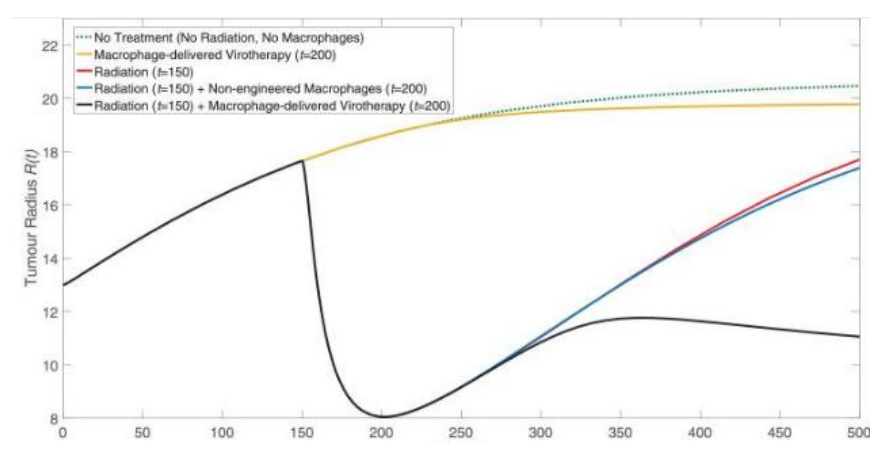

Fig. 7 The Solution of Given Equations
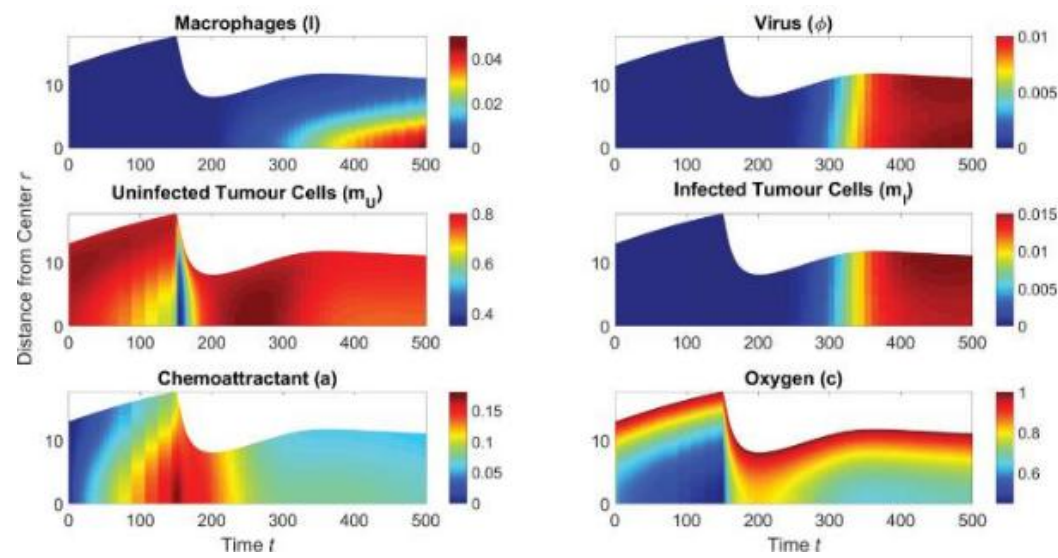

Fig. 8 Series of Plots Corresponding to the above Black Curve

As the figure shows, the initial decrease in MU, non-infectious microbial tumors, is caused by radiotherapy, which reduces the tumor size because the remaining cells are concentrated in a smaller tumor mass. This decrease in tumor size makes it better for oxygen to be absorbed into the tumor center, and cell death due to radiotherapy increases chemotherapy. Later, as the tumor radius increases, the macrophage infiltration rate decreases, as macrophages are less prone to transfer to obtain the nucleus.

\section{Conclusions}

The present study investigates the ability of MRV to infect hypoxia-like cells. This paper also investigates the simple involvement of each MRV protein in the inhibition of HIF-1 $\alpha$, and the proteins obtained from $\mu 1$ and $\mu 2$ can regulate HIF-1 $\alpha$ freely. Prostate Cancer Interdependence (PCA) on androgens is performed for growth and survival by hormone therapy, where androgen deprivation is used as an appropriate treatment. However, initially successful, this androgen-related PCA develops into a dependent PCA and rogenind, where hypoxia has been shown to play a critical role. And hence, limit the harmful effects of hypoxia, which may be forced during androgen treatment and may lead to the development of androgen-independent prostate tumor cells. Activities containing Akt activity that enhances AR function despite low androgen levels and inhibits translation resulting in decreased protein and AR activity that are dependent on PCP-independent PCP for continued growth and development of PCA, also proving PSA levels that also is adjusted, inhibited. In addition, this paper also simulates a tumor modulator framework based on mixed theory. While more numerical and experimental work is needed for the exact parameter of the model, our results prove that microclasts should be introduced immediately after radiotherapy to provide the maximum treatment.

\section{References}

[1]. Bray S.J. 2006, Notch signalling: a simple pathway becomes complex. Nat. Rev. Mol. Cell. Biol 2006; 7: 678-689. Sainson R. C. A and Harris A. L. hypoxiaregulated differentiation: lets step it up a Notch. Notch. Trends Mol. Med; 12(4): 141-143.

[2]. Crivelli J.J., Földes J., Kim P.S., Wares J.R., 2012, A mathematical model for cell cycle-specific cancer virotherapy. J. Biol. Dyn. ;6:104-120.

[3]. Eftimie R., Eftimie G., 2018, Tumour-associated macrophages and oncolytic virotherapies: a mathematical investigation into a complex dynamics. Lett. Biomathem.;5:70-99.

[4]. Fruehauf J. P and Meyskens F. L, Jr., 2007, Reactive oxygen species: A breath of life or death? Clin. Cancer Res; $13: 789-794$.

[5]. Gustafsson M. V, et al., 2005, hypoxia requires Notch signalling to maintain the undifferentiated cell state. Dev. Cell; 9: 617-628.

[6]. Hensley K, Robinson K. A, Gabbita S. P, Salsman S and Floyd R. A., 2000, Reactive oxygen species, cell signalling, and cell injury. Free Radic. Biol. Med; 28:1456-1462.

[7]. Hochachka P. W, Buc, L. T, Doll C. J and Land S. C., 1996, Unifying theory of hypoxia tolerance: molecular/ metabolic defense and rescue mechanisms for surviving oxygen lack. Proc. Natl. Acad. Sci. U.S.A; 93: 9493- 9498.

[8]. Kim J. W, Tchernyshyov I, Semenza G. L and Dang C. V., 2006, HIF-1-mediated expression of pyruvate dehydrogenase kinase: A metabolic switch required for cellular adaptation to hypoxia. Cell Metab; 3: 177-185. 
[9]. Maithili Sharan and Aleksander S. Popelj, 2002. "A Compartmental Model for Oxygen Transport in Brain Microcirculation in the Presence of Blood Substitutes".

[10]. Mekhail K, Gunaratnam L, Bonicalzi M. E and Lee S., 2004. HIF activation by PH-dependent nucleolar sequestration of VHL. Nat. Cell Biol; 6: 642-647.

[11]. Moody W, Jr., 1984. Effects of intracellular H+ on the electrical properties of excitable cells. Annu. Rev. Neurosci; 7: 257-278.

[12]. Owen M.R., Byrne H.M., Lewis C.E., 2004 Mathematical modelling of the use of macrophages as vehicles for drug delivery to hypoxic tumour sites. J. Theor. Biol.; 226:377-391

[13]. Rodkiewicz, C.M., Sinha, P. Kennedy, J.S., 1990. "On the application of a Constitutive equation for the whole human blood", Journal of Biomechanical Engineering, 113, 198-206.

[14]. Sauer H, Wartenberg M and Hescheler J., 2001. Reactive oxygen species as intracellular messengers during cell growth and differentiation. Cell Physiol. Biochem; 11: 173-186.

[15]. Semenza G. L, Roth P. H, Fang H. M and Wang G. L., 1994. Transcriptional regulation of genes encoding glycolytic enzymes by hypoxiainducible factor 1. J. Biol. Chem; 269: 23757-23763.

[16]. Sharma, G.C.; Jain, M. and Kumar, Anil, 2001. "Finite element Galerkin"s approach for a computational study of arterial flow" International Journal of Applied Mathematical and Mechanics, China (English Edition), 22(9), 1012-1018.

[17]. Silver I. A and Erecinska M. J., 1992. Reported on the ion homeostasis in rat brain hippocam- pus during recovery from short-term transient. Cereb. Blood Flow Metab; 12: 759-772.

[18]. Thannickal V. J and Fanburg B. L., 2000. Heme oxygenase: colors of defense against cellular stress. Am. J. Physiol Lung Cell Mol. Physiol; 279: L1005-L1028.

[19]. Wang G. L and Semenza G. L., 1993. General involvement of hypoxia-inducible factor 1 in transcriptional response to hypoxia. Proc. Natl. Acad. Sci. U.S.A; 90 4304-4308.

[20]. Yao H and Haddad G. G., 2004. Calcium and PH homeostasis in neurons during hypoxia and ischemia. Cell Calcium; 36: 247-255. 